\title{
El sismo del 21 de enero de 2003 en Colima: estudio de la percepción social para la gestión del riesgo de desastres
}

\author{
Earthquake of 21 January 2003 in Colima, Mexico: \\ Study of Social Perception for Disaster Risk Management
}

Karla Nayeli De Dios Dávalos ${ }^{1}$ y Juan Manuel Rodríguez Esteves ${ }^{2}$

\section{Resumen}

El objetivo de este artículo es analizar algunos componentes de la percepción social del riesgo sísmico en la ciudad de Colima; en particular se consideró la zona centro por ser el espacio donde se concentraron los daños relacionados con el sismo del 21 de enero de 2003. Para comprender la percepción del riesgo, se aplicó un cuestionario a 322 residentes de la zona de estudio bajo la técnica de redes semánticas naturales, mediante la cual se determinó que existen cinco categorías de significados: las creencias y emociones, características del fenómeno, resultados postdesastre, componentes del desastre y respuesta a la emergencia. Entre las principales conclusiones a las que se llegó destaca que la percepción de la población acerca del riesgo está constituida por una serie de factores que van desde la escala individual hasta la social. Una de las principales limitaciones de la investigación, aunque al mismo tiempo puede ser una fortaleza, radica en su corte temporal ya que, a 18 años de ocurrido el sismo, el recuerdo de los acontecimientos puede ser modificado por el olvido.

Palabras clave: sismo; riesgo; desastre; percepción del riesgo; redes semánticas.

\footnotetext{
1 Autora de correspondencia. Maestría en Administración Integral del Ambiente por El Colegio de la Frontera Norte (COLEF) y el Centro de Investigación Científica y Educación Superior de Ensenada (CICESE), México. Consultora independiente. Líneas de interés: percepción del riesgo, comunicación del riesgo, riesgo de desastres. ORCID: https://orcid.org/0000-0003-4478-3556. Correo electrónico: karla_nayeli11@hotmail.com

${ }^{2}$ Doctorado en Ciencias Sociales con Especialidad en Antropología Social por el Centro de Investigaciones y Estudios Superiores en Antropología Social (CIESAS), Unidad Occidente, México. Investigador de El Colegio de la Frontera Norte (COLEF), sede Tijuana, México. Líneas de interés: geografía del riesgo, antropología de los desastres, problemas ambientales. ORCID: https:/orcid.org/0000-0002-9022-9655. Correo electrónico: jesteves@colef.mx
} 


\begin{abstract}
This article's objective is to analyze some components of the social perception of seismic risk in the city of Colima, Mexico. In particular, we considered the central zone of this city because it concentrated the damages related to the earthquake of January 21, 2003. To understand the perception of risk, we applied a questionnaire to 322 residents of the study area under the technique of natural semantic networks, through which we determined that there are five categories of meanings: beliefs and emotions, characteristics of the phenomenon, post-disaster results, disaster components, and emergency response. Among the main conclusions reached, it stands out that the population's perception of risk is composed of a series of factors ranging from the individual to the social scale. One of the main limitations of the research, although at the same time it can be a strength, lies in its temporal cutoff since, 18 years after the earthquake occurred, the memory of the events can be modified by oblivion.
\end{abstract}

Keywords: disaster; earthquake; risk; risk perception; semantic networks.

\title{
Introducción
}

El territorio mexicano es una región expuesta a la ocurrencia de sismos de gran magnitud. Los sismos son fenómenos geológicos recurrentes, ya que para el periodo de 1999 a 2019 se registraron 82 eventos con magnitudes que van de 6.0 a 9.9 (SSN, 2020). Los daños asociados a estos sismos estuvieron relacionados con la pérdida de infraestructura como carreteras, líneas de transmisión, canales de riego, viviendas, etcétera, cuyo monto ascendió a \$77 398.8 millones de pesos (SEGOB, 2019). El estado de Colima no ha sido la excepción, ya que a través de los años se han registrado sismos que han ocasionado severos daños, eventos de particular relevancia cuando las afectaciones se presentan en contextos de vulnerabilidad. Los daños y pérdidas, así como la afectación de la vida cotidiana de las personas, permite reflexionar acerca de los múltiples riesgos a los que está expuesta la población colimense y, sobre todo, la percepción que tienen sobre éstos (Cuevas y Seefoo, 2005).

La actividad de las principales placas tectónicas que influyen en el país, como la del Pacífico, Norteamericana, Rivera y Cocos, han sido la fuente principal de sismos que han afectado los estados ubicados en la costa del Océano Pacífico, entre los que se encuentra Colima (Cenapred, 2014a). Con base en la información proporcionada por la serie Impacto socioeconómico de los principales desastres ocurridos en la República mexicana, elaborada por el Centro Nacional de Prevención de Desastres (Cenapred), se elaboró el Cuadro 1 , donde se presentan los principales impactos asociados a sismos desde 1985 hasta 2018. 


\section{Cuadro 1. Principales daños relacionados con sismos de magnitud mayor a 7 en México (1985-2018)}

\begin{tabular}{|c|c|c|c|c|c|}
\hline Entidad federativa & Año & Día/mes & Magnitud & Muertes & Total de daños \\
\hline Ciudad de México & 1985 & 19 de septiembre & 8.1 & 6043 & 4104 \\
\hline Guerrero y Oaxaca & 1995 & 14 de septiembre & 7.3 & N.D. & 21 \\
\hline Michoacán, Colima y Jalisco & 1995 & 9 de octubre & 8.0 & 30 & $\mathrm{~N} / \mathrm{D}$ \\
\hline Oaxaca & 1999 & 30 de septiembre & 7.5 & 35 & 155 \\
\hline Colima, Jalisco y Michoacán & 2003 & 21 de enero & 7.6 & 26 & 1290 \\
\hline Baja California & 2010 & 4 de abril & 7.2 & 2 & 8644 \\
\hline Guerrero y Oaxaca & 2012 & 20 de marzo & 7.4 & 2 & 1419 \\
\hline Ciudad de México & 2017 & 19 de septiembre & 7.1 & 228 & 43040 \\
\hline Oaxaca & 2017 & 7 de septiembre & 8.2 & 79 & 10322 \\
\hline Morelos & 2017 & 19 de septiembre & 7.1 & 74 & 7322 \\
\hline Puebla & 2017 & 19 de septiembre & 7.1 & 45 & 4495 \\
\hline Chiapas & 2017 & 7 de septiembre & 8.2 & 16 & 8861 \\
\hline Edo. de México & 2017 & 19 de septiembre & 7.1 & 15 & 3929 \\
\hline Guerrero & 2017 & 19 de septiembre & 7.1 & 6 & 1839 \\
\hline Oaxaca & 2018 & 16 de febrero & 7.2 & 0 & 1425 \\
\hline
\end{tabular}

Nota: hasta los sismos de 1999, el total de daños se calculó en millones de dólares, posteriormente son millones de pesos.

Fuente: elaboración propia con datos de Cenapred, varios años. Serie: Impacto socioeconómico de los principales desastres ocurridos en la República mexicana. Recuperado de https://www.gob.mx/cenapred

El sismo que tuvo una influencia determinante en la respuesta del gobierno frente al desastre fue el registrado el 19 de septiembre de 1985, al crearse el Sistema Nacional de Protección Civil (Sinaproc) (Garza, 1998; AlcántaraAyala et al., 2019), el cual ha sido un referente para otros países de América Latina (Banco Mundial, 2012). Los sismos que más han afectado al país han sido los de magnitud 7 y mayores, aunque el monto total de daños están referidos sólo a los recursos aplicados por el Fondo de Desastres Naturales (Fonden); recursos provenientes de los gobiernos federal y estatales que se aplican a infraestructura pública, pero no reflejan el total de daños reales. De igual forma, los sismos registrados durante 2017, en particular el ocurrido en Ciudad de México, puso en marcha estrategias sociales para atender los efectos del sismo, aunque ya se habían visto en 1985, pero esta vez a través de la acción de grupos de voluntarios utilizando herramientas digitales como las redes sociales, aportando de manera significativa a la respuesta ante el desastre (Anzaldo et al., 2020).

Los daños relacionados a sismos no sólo están asociados a la magnitud del evento natural, sino que es necesario entender las causas sociales, ambientales y económicas ya que, como se puede ver en el Cuadro 1, el sismo magnitud 8.2 del 7 de septiembre de 2017 en Oaxaca, reportó menos daños 
que el ocurrido en Ciudad de México el día 19, cuya magnitud fue de 7.1. Para entender cómo los desastres pueden llegar a impactar a amplios sectores de la población, se deben considerar algunos términos como la amenaza, vulnerabilidad, riesgo y el propio desastre, que en ocasiones puede ser entendido como emergencia.

De acuerdo con el Informe del grupo de trabajo de expertos relacionados con la reducción del riesgo de desastres, de la Organización de las Naciones Unidas (2016: 19), una amenaza es un "fenómeno o actividad humana que puede ocasionar muertes, lesiones u otros efectos en la salud, daños a los bienes, disrupciones sociales y económicas o daños ambientales”. Las amenazas están relacionadas con su origen, por ejemplo, las amenazas naturales se asocian con los fenómenos naturales intensos, mientras que las amenazas antropógenas tienen un origen humano; la combinación de ambas suelen denominarse amenazas socionaturales. De igual forma, la vulnerabilidad se refiere a las "condiciones determinadas por factores físicos, sociales, económicos y ambientales que aumentan la susceptibilidad de una persona o una comunidad a los efectos de las amenazas" (ONU, 2016: 25).

El riesgo de desastres hace referencia a la "posibilidad de que se produzcan muertes, lesiones o destrucción y daños en bienes en una sociedad o una comunidad en un periodo de tiempo concreto, determinados de forma probabilística como una función de la amenaza, la exposición, la vulnerabilidad y la capacidad" (ONU, 2016: 15). Por lo tanto, el desastre es la "disrupción grave del funcionamiento de una comunidad o sociedad en cualquier escala debida a fenómenos peligrosos que interaccionan con las condiciones de exposición, vulnerabilidad y capacidad, ocasionando uno o más de los siguientes: pérdidas e impactos humanos, materiales, económicos y ambientales" (ONU, 2016: 13). En ocasiones, el término de desastre se utiliza de manera indistinta con el de emergencia, sin embargo, este último no suele provocar una disrupción grave del funcionamiento de una comunidad o sociedad.

Los términos anteriormente presentados, permiten contextualizar el enfoque de la percepción del riesgo, elemento central de esta investigación. La percepción del riesgo puede ser abordada desde dos paradigmas: el psicométrico y el sociocultural. El primero de ellos está representado por Paul Slovic (2000), que se enfoca en el campo psicosocial y ha sido ampliamente estudiado por la psicología, buscando una comprensión de la percepción individual y colectiva de un fenómeno en específico. Aunque el riesgo es el mismo para una sociedad, la manera en que es percibido por sus miembros va a depender de diferentes factores como el control, la confianza que se tiene en la comunidad científica, el beneficio que el riesgo puede proporcionar a la población, entre otros. Conocer los riesgos debe ser relevante, ya que de eso dependerá la manera de actuar frente a ese riesgo (Slovic, 2000). De igual modo, Slovic (1987) destaca que el conocimiento de la percepción del riesgo es, en ocasiones, mayor que el conocimiento de los científicos, ya que permite profundizar en las preocupaciones de la población, 
las cuales suelen ser ignoradas en las evaluaciones del riesgo realizadas por la comunidad científica.

El paradigma sociocultural lo representa Mary Douglas (1987), quien propone tres recomendaciones sobre la percepción del riesgo, es decir, las variaciones sociales de las decisiones, el modelo probabilístico respecto al riesgo y el peso moral de esas decisiones. Estos tres principios, permiten entender el riesgo desde el enfoque cultural, a través de la cual el riesgo es clasificado según el grupo que lo interpreta. En este sentido, Douglas y Wildavsky (1982) consideraron que los procesos sociales y culturales, moldean la visión de una comunidad frente a un riesgo determinado, por lo tanto, la percepción hacia un riesgo puede tener significados diferentes para ciertos grupos.

En el estudio de la percepción del riesgo, se establece que "los individuos tienen un sentido fuerte, pero injustificado, de inmunidad subjetiva" (Douglas, 1996: 57), es decir, cuando el individuo realiza actividades comunes, tiende a minimizar el riesgo de obtener un mal resultado. Por ejemplo, al momento de conducir un vehículo, algunas personas tienden a minimizar el riesgo de sufrir un accidente de tráfico, ya que su atención está en llegar a tiempo a su destino. De igual modo, un elemento que influye sobre la percepción es la memoria, pero algunos seres humanos no llegan a pensar racionalmente, ya que pueden tener una memoria débil (Douglas, 1996). Esta autora profundiza en el tema y afirma que "dado que en el caso de la memoria humana lo que está almacenado y es recuperable depende del control de la atención (...) la cuestión está en enfocar el problema desde el ángulo adecuado y centrar la atención en la codificación que el individuo realiza de la experiencia social" (Douglas, 1996: 59). Con base en ello, en esta investigación se formuló una pregunta clave para recuperar de la memoria de los encuestados sus experiencias y actitudes acerca del riesgo sísmico.

Los factores que intervienen en la percepción al riesgo sísmico son variados, complejos y actúan a diferentes escalas del conocimiento individual y social. De esta forma, se seleccionó el riesgo sísmico para identificar si prevalece en los afectados el recuerdo u olvido de los efectos del sismo del 21 de enero de 2003, además de conocer sus reacciones, a partir de un evento natural que desencadenó el desastre, lo que puede ofrecer elementos que se deberían considerar en la gestión del riesgo y mitigar daños futuros. Para ello, se planteó la pregunta ¿cuáles fueron las respuestas y actitudes tomadas por los afectados durante el sismo de 2003? Con base en este planteamiento, se partió del supuesto de que los individuos mantienen en la memoria los recuerdos en función a la magnitud de resultados negativos para ellos y sus familias, es decir, cuando se experimentan daños de gran magnitud, los individuos mantienen el recuerdo por más tiempo. Por lo tanto, el objetivo fue analizar los factores que intervienen en la percepción del riesgo sísmico entre los habitantes de la zona centro de la ciudad de Colima a partir del evento registrado el 21 de enero de 2003. 
La estructura del artículo se organizó en dos partes. En la primera sección, se desarrolla la estrategia metodológica, donde se explican los criterios para delimitar la zona de estudio, las fuentes de información utilizadas, así como la primera campaña de reconocimiento de campo. En la segunda sección, se presentan los resultados y la discusión de la información obtenida en campo, de manera particular se explican los aspectos generales de los encuestados, las respuestas al sismo del 21 de enero de 2003 y el análisis de la percepción del riesgo sísmico. Por último, se presentan las conclusiones, donde se destacan algunos factores que intervienen en la percepción del riesgo sísmico de los habitantes en la zona de estudio.

\section{Estrategia metodológica}

El manejo y análisis de la información se basaron en un enfoque mixto, ya que se emplearon técnicas cualitativas y cuantitativas. El enfoque cualitativo permitió caracterizar y comprender los procesos que influyen en la percepción del riesgo; para ello se procedió a revisar bibliografía especializada e investigaciones sobre el tema, además de periódicos que cubrieron el evento para identificar las zonas más afectadas. El método cualitativo, según Hernández y colaboradores (2014: 8), "se basa en métodos de recolección de datos no estandarizados ni predeterminados completamente". Asimismo, Patton (2011, citada en Hernández et al., 2014: 9) describe los datos cualitativos como "descripciones detalladas de situaciones, eventos, personas, interacciones, conductas observadas y sus manifestaciones".

Por otro lado, usando un método cuantitativo, según Hernández y colaboradores (2014: 6) "se intenta generalizar los resultados encontrados en un grupo o segmento (muestra) a una colectividad mayor (universo o población)". En este caso, para la recolección de datos se emplearon diferentes técnicas metodológicas: trabajo de campo, consulta de bases de datos ubicadas en bibliotecas locales, y se elaboró un cuestionario estructurado de preguntas tanto abiertas como de opción múltiple dirigido a la población afectada por el sismo de 2003. A continuación, se describen tanto la selección del área de estudio como las herramientas y técnicas metodológicas empleadas.

\section{La zona de estudio, fuentes de información y reconocimiento en campo}

La ciudad de Colima se encuentra ubicada en la provincia del Eje neovolcánico, y el representante más emblemático es el volcán de Colima, localizado en el extremo norte del estado. La ciudad ocupa una extensa llanura aluvial, caracterizada por ser una zona sin elevaciones importantes y el terreno está conformado por material no consolidado, es decir, transportado y depositado por corrientes de agua (Ayuntamiento de Colima, 
2012). La regionalización sísmica de México, ubica a Colima en la categoría $\mathrm{D}$, la más alta debido a su sismicidad, donde la aceleración del terreno puede ser mayor a $70 \%$ del valor de la gravedad (g). La presencia de un suelo aluvial, más la alta sismicidad, puede amplificar la intensidad de los sismos. En Colima, los sismos se generan principalmente por la interacción de las placas de Cocos y Rivera en el Océano Pacífico y la placa continental de Norteamérica (Cenapred, 2014a; Cenapred, 2004). Al respecto, destaca el sismo del 3 de junio de 1932 (magnitud de 8.2), que provocó un tsunami de dos metros en las costas de Manzanillo. De igual forma, el sismo del 30 de enero de 1973 (magnitud 7.6), también provocó olas de $1.1 \mathrm{~m}$ en la costa (Cenapred, 2003: 304). En años recientes, se han registrado eventos a los que han estado relacionados cuantiosos daños como el del 9 de octubre de 1995 y, más recientemente, el del 21 de enero de 2003 (Cuadro 1).

El sismo del 21 de enero de 2003 que afectó a Colima, denominado sismo de Tecomán, se registró a las 20:06 h, 21 personas fallecieron por situaciones relacionadas con el movimiento telúrico y se instalaron nueve albergues donde fueron atendidas 2 mil personas (Cenapred, 2003). El sismo tuvo una magnitud de 7.6, con una duración mayor a un minuto, causando graves destrozos en las ciudades de Colima, Villa de Álvarez, Tecomán, Manzanillo y Coquimatlán, en el estado de Colima. Los reportes oficiales informaron, además del número de muertes, más de 15 mil casas dañadas (Cortes, Badillo y Trejo, 2003; SEDATU, 2014).

Los daños en el estado de Colima ascendieron, en millones de pesos, a $\$ 493.1$ en infraestructura social, $\$ 180.4$ en infraestructura económica, $\$ 244.2$ en el sector productivo y $\$ 156.3$ en edificios públicos y patrimonio cultural (Cenapred, 2004). El sismo provocó mayores daños en las viviendas, particularmente en las construidas con adobe, de mampostería no reforzada y de concreto de mala calidad. La ciudad de Colima fue la más afectada en cuanto al número de viviendas dañadas, con 5874 viviendas, y el municipio contiguo, Villa de Álvarez, registró 2649 viviendas con algún tipo de daño. La concentración de viviendas dañadas en la zona de estudio se ubicó en el centro de la ciudad (Cenapred, 2004).

De acuerdo con el Atlas de Peligros y Riesgos en el Estado de Colima (Gobierno del Estado de Colima, 2015), en el que se realizó una evaluación de las viviendas en 2420 manzanas en la ciudad de Colima entre 2008 y 2014, se encontró que 300 (12\%) son del Tipo 1; 1437 (60\%) son del Tipo 2 y 683 son del Tipo 3 (28\%). Los tipos de vivienda están en función del material y método de construcción; así las de Tipo 1 son de buena calidad, ya que se usaron ladrillos o concreto, son de dos niveles y no tienen más de 20 años de haber sido construidas. Las de Tipo 2 son de uno o dos pisos y poseen materiales de mediana a baja calidad, como es el caso de las construcciones de interés social. Por su parte, las de Tipo 3, o de mala calidad, son construcciones antiguas realizadas con adobe, y carecen de elementos estructurales como castillos, dalas de cerramiento con cubierta de teja o láminas sin anclajes como las encontradas en las colonias populares 
(Gobierno del Estado de Colima, 2015). Esta información fue un insumo para el cálculo de las categorías presentadas en la Figura 1.

Figura 1. Microzonificación de riesgo sísmico de la zona metropolitana de Colima según vulnerabilidad sísmica de la vivienda

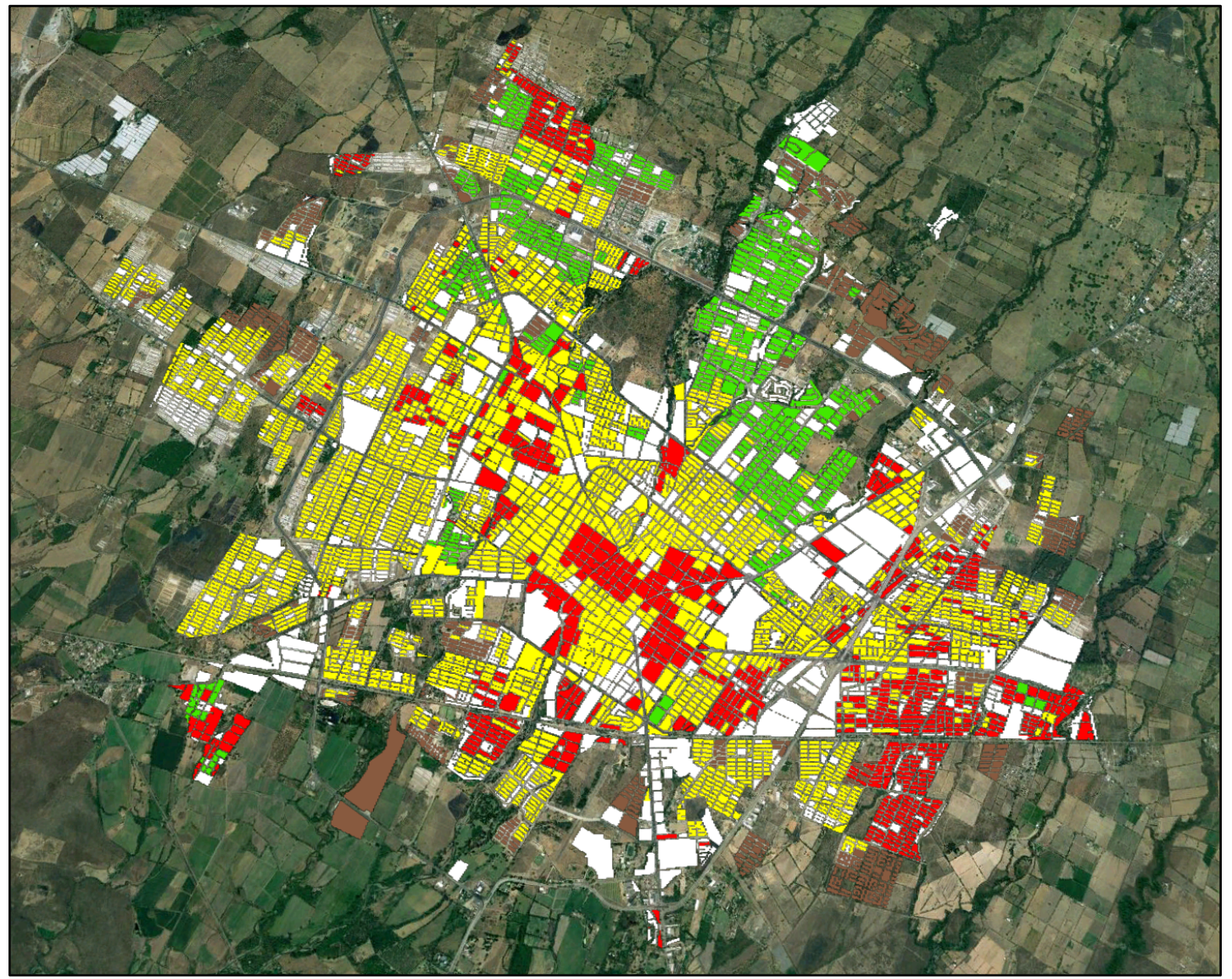

Simbología: zonas color rojo: riesgo alto; zonas color amarillo: riesgo medio; zonas color verde: riesgo bajo; zonas color café: manzanas no construidas; zonas color blanco: edificios públicos.

Fuente: Gobierno del Estado de Colima (2015) y Cenapred (2020).

Con base en la Figura 1, se observa que la ciudad presenta un diseño concéntrico, es decir, en la parte central, o centro histórico, se concentran las viviendas Tipo 3, construidas con adobe y otros materiales no reforzados. Por su parte, las viviendas Tipo 1 , con materiales reforzados como concreto y ladrillo, se ubican en la zona norte, coincidiendo con los nuevos fraccionamientos y un promedio de construcción de 20 años (Gobierno del Estado de Colima, 2015). Con base en esta información, el centro urbano resultó ser una zona potencial para aplicar el cuestionario, ya que concentra las viviendas construidas con adobe y, por lo tanto, es una de zonas con mayor riesgo. 
Por lo que respecta al levantamiento de información, se realizó una campaña de trabajo de campo durante los meses de julio y agosto de 2019 para recopilar información bibliográfica y hemerográfica. En esta etapa, se consultó información para conocer el contexto socioambiental e histórico de la zona de estudio, con especial énfasis en los sismos. Las fuentes de información se ubicaron en las bibliotecas de la Universidad de Colima, de Ciencias, Ciencias de la Salud, así como en el Archivo Histórico de Colima. Las consultas hemerográficas se realizaron en los periódicos El Noticiero de Colima, Ecos de la Costa y Diario de Colima. De igual modo, se realizaron entrevistas al Dr. Vyacheslav M. Zobin y al Dr. Raúl Arámbula Mendoza, expertos de la Universidad de Colima y con representantes de las áreas de Protección Civil, el director estatal, Ricardo Urzúa y el director municipal, Abel Meneses, para conocer su opinión y obtener más información.

Los resultados de esta etapa arrojaron que sería la zona centro de la ciudad de Colima, el lugar donde se aplicaría el cuestionario, por ser la zona que registró mayores daños relacionados con el sismo del 21 de enero de 2003, en particular, se seleccionaron las colonias San Isidro, municipio de Villa de Álvarez, y la zona Centro, municipio de Colima. Esto también tiene congruencia con la información presentada en la Figura 1, donde gran parte de las zonas de alto riesgo, viviendas Tipo 3 (adobe), se concentran en esta zona. Adicionalmente, se realizó una prueba piloto del cuestionario para hacer ajustes al listado de preguntas y preparar la segunda campaña de trabajo de campo.

\section{Diseño y aplicación del cuestionario}

La segunda etapa de trabajo de campo se realizó durante enero y febrero de 2020 para aplicar el cuestionario y el procesamiento de información. Se elaboró un cuestionario con 24 reactivos y se aplicó a 322 habitantes de la zona centro de la ciudad de Colima. La selección del universo y la muestra se diseñó con base en el número de habitantes en dos colonias de la zona centro y, como condición previa, los encuestados tenían que ser mayores de 21 años y haber experimentado el sismo de enero de 2003. Esto pudiera representar una limitante para el estudio, ya que no es el mismo recuerdo de una persona de cuatro años al momento de la manifestación del sismo (con 21 años al aplicar el cuestionario), que una persona con 18 años durante el sismo de 2003 (35 años en la actualidad), sin embargo, el $75 \%$ de los entrevistados tenían entre 30 y 85 años o más. La población total fue de 1 963 habitantes, correspondiendo a la colonia Centro de Colima 742 habitantes, y para la colonia San Isidro, Villa de Álvarez, 1221 habitantes (INEGI, 2015). Para el cálculo de los tamaños de la muestra se utilizó la fórmula propuesta por Romo (1998: 55). 


$$
n=\frac{\mathrm{Npq}}{\frac{M e^{2}}{N c^{2}}(\mathrm{~N}-1)+p q}
$$

Donde:

$\mathrm{n}=$ tamaño de la muestraN = tamaño del universo

$\mathrm{p}=$ probabilidad de ocurrencia (.5)

$\mathrm{q}=$ Probabilidad de no ocurrencia $(1-\mathrm{p}=.5)$

$\mathrm{Me}^{2}=+/-5 \%$ de margen de error (.5)

$\mathrm{Ne}^{2}=$ Valor z que determina el área de probabilidad buscada (1.96) .4

De esta forma, sustituyendo los valores de la fórmula se obtuvo el siguiente resultado:

$$
\begin{gathered}
n=\frac{1963(0.5)(0.5)}{\frac{.05^{2}}{1.96^{2}}(1963-1)+(0.5)(0.5)} \\
n=\frac{490.75}{\frac{0.0025}{3.8416}(1962)+0.25} \\
n=\frac{490.75}{0.00065(1962)+0.25} \\
n=\frac{490.75}{1.5253}=\mathbf{3 2 1 . 7 3 9}
\end{gathered}
$$

Por lo tanto, el instrumento se aplicó a 322 habitantes de las colonias Centro y San Isidro, en la ciudad de Colima, visitando cada una de las viviendas y aplicando el cuestionario sólo en los casos que se cumplieran las dos condiciones previas, es decir, ser mayor de 21 años y haber experimentado el sismo del 21 de enero de 2003. La encuesta estuvo conformada por las siguientes secciones:

a. Datos generales: edad, sexo, lugar de nacimiento y residencia, ocupación, escolaridad, estado civil, así como el tiempo que tenían residiendo en la ciudad para asegurar que vivieron el sismo seleccionado como caso de estudio.

b. Conocimiento previo: se incluyó la palabra "estímulo respecto a los sismos", donde los entrevistados mencionaron cinco palabras 
definidoras, con la intención de identificar elementos sobre el conocimiento acerca de sismos.

c. Experiencia ante sismos: preguntas de opción múltiple sobre su experiencia del sismo del 2003, qué hacía en ese momento, qué hizo durante y después del mismo.

d. Prevención: este apartado se diseñó con preguntas de opción múltiple acerca de las medidas preventivas en caso de sismo y cómo las llevaban a cabo, además de una pregunta abierta para conocer las medidas de prevención que la persona pueda emplear.

e. Responsabilidad: la sección final estuvo compuesta de preguntas para conocer quién tiene la responsabilidad en cuanto a seguridad al momento de la ocurrencia de un sismo, así como una pregunta abierta para saber qué consideran que hace falta para evitar daños mayores al momento de un sismo.

De igual modo, se incluyó un apartado para conocer la percepción del riesgo sísmico mediante la técnica de redes semánticas naturales. Esta técnica permite observar los procesos de representación colectiva, así como sus significados, también "aporta elementos teóricos y empíricos para generar conocimiento sobre la percepción como componente del conocimiento, en donde el sujeto interactúa con el mundo objetivo al percibirlo, por lo que la percepción está ligada al lenguaje" (Arellano et al., 2012: 169). Esta técnica es definida por Vargas et al. (2014) como palabras que son recuperadas mediante la memoria, por ello "se trata de las ideas y conceptos que las personas construyen acerca de cualquier objeto, tema o situación de su cotidianidad y son expresados también en términos coloquiales" (Vargas et al., 2014: 9). Por su parte, Zermeño y colaboradores (2005: 310) establecieron que:

Las taxonomías obtenidas son generadas de manera directa de la memoria semántica del sujeto, y el orden otorgado va de acuerdo con su escala de valores y percepciones. Cuando se le pide al sujeto que mencione las palabras que definen al concepto [palabra estímulo], éste hurga en su memoria y selecciona aquellas que asume más relacionadas; en este sentido, la elección es resultado de un proceso subjetivo de representarse al mundo. Así, la mediación del investigador se limita a la estimulación, no interviene durante la búsqueda y la selección de las palabras con las que el sujeto define al objeto.

La forma de diseñar este instrumento constó de tres fases. En primer lugar, se seleccionó una o más palabras estímulo, a fin de conocer el significado asignado por los sujetos de estudio al evento sísmico. En segundo lugar, se pidió a los sujetos que intentaran definir la palabra estímulo mediante cinco 
palabras sueltas; una vez que los individuos realizaron la lista de palabras, se les pidió que las jerarquizaran según la importancia que tienen las palabras en relación con la palabra estímulo, asignándole un valor del uno al cinco, donde uno es el más importante y así sucesivamente. En tercer lugar, se realizó el vaciado de la información, se construyó una base de datos con todas las palabras mencionadas por los individuos, así como casillas del lado derecho para registrar la jerarquía que se le asignó a cada una. Finalmente, una vez que se obtuvieron todas las palabras definidoras, se calcularon los valores de la red semántica (Vera et al., 2005, citado en Hinojosa, 2008).

Para analizar los datos obtenidos mediante esta técnica, se obtuvieron los cuatro valores principales: valor $\mathrm{J}$, valor $\mathrm{M}$, valor $\mathrm{G}$, conjunto SAM y valor FMG. El valor $J$ equivale al total de las palabras diferentes que se mencionaron. El valor $\mathrm{M}$ es el valor semántico de las palabras, el cual es asignado por el encuestado según su criterio. El valor $G$, indica la dispersión de las palabras dentro de la red semántica. El conjunto SAM es el conjunto de las palabras que obtuvieron los mayores puntajes. Finalmente, el valor FMG indica la distancia que existe entre cada palabra definitoria con relación a las que se encuentran en los primeros lugares (Arellano et al., 2012).

Para la obtención de esos valores, se construyó una base de datos en la hoja de cálculo Excel, donde se capturaron las palabras definidoras asociadas a la palabra "sismo". Posteriormente, se le añadió a cada palabra el valor semántico asignado por el encuestado; los valores iban del 1 al 5 , siendo el 1 el más importante. De esta forma, se obtuvo información que permite configurar algunos elementos que determinan la percepción social del riesgo sísmico en la zona de estudio, destacando cinco categorías de significados según valor semántico, las cuales se abordarán en la siguiente sección.

\section{Resultados y discusión}

\section{Aspectos generales}

El uso de la estadística descriptiva, permitió identificar que del total de los encuestados, $45 \%$ fue población masculina y $55 \%$ femenina. De esta forma, se observó que los grupos de edad estuvieron en los rangos de 30 y 40 años (28\%), 21 a 29 años (25\%), 41 a 51 años (14\%), 52 a 62 años (14\%), 63 a 73 años (11\%), 74 a $84(6 \%)$ y 85 años o más (2\%). Un dato a destacar es que el $75 \%$ de la población tuvo una edad mayor a los 30 años al momento de la encuesta, y la proporción de personas entre 21 y 29 años (25\%) se debió a que el levantamiento de la información se realizó en el periodo vacacional de invierno de 2020.

Por lo que respecta al grado de estudios de la población encuestada, predominó el nivel profesional (41\%), seguido del nivel preparatoria (25\%), 
secundaria (14\%), primaria (13\%) y maestría (7\%). En esta variable, destaca la proporción de mujeres con escolaridad profesional o maestría, ya que fue de $49 \%$, mientras que en hombres fue de $46 \%$. Es importante señalar el nivel de escolaridad de los encuestados, ya que esto pudo influir en el conocimiento objetivo acerca del sismo, y que ofrecieran una explicación más técnica sobre el fenómeno experimentado.

\section{El sismo del 21 de enero de 2003}

El elemento central de la investigación es el sismo del 21 de enero de 2003, por ello, se hizo mención específica del evento para traer a la memoria de los encuestados sus experiencias, aunque esto representó un esfuerzo para el $25 \%$ de la población (con 21 a 29 años durante la encuesta), que al momento del sismo contaban con cuatro o cinco años. De esta forma, el $100 \%$ de las personas encuestadas declararon haber experimentado de alguna manera los efectos de un sismo.

En cuanto a los sismos ocurridos en la zona de estudio, el $54 \%$ de la muestra sólo experimentó el sismo del 21 de enero de 2003, el 26 \% también experimentó el de 1995, el 16 \% recordó el de 1985, y sólo 4 \% experimentó también el sismo de 1973. Como se observa, el número de experiencias frente a sismos va disminuyendo conforme más antiguo sea el evento, lo cual está asociado con la edad de los encuestados y, quizás, con el olvido. Cabe mencionar que varios encuestados ignoraban algunos de los sismos anteriores al de 2003, lo que se explica por su rango de edad. En este sentido, la memoria colectiva de la sociedad se extiende hasta donde llega la memoria de los grupos que la componen, por lo tanto, se soporta en un grupo limitado en el tiempo y en el espacio (Halbwachs, 1968, citado en Bracamontes, 2015).

La Gráfica 1 muestra las reacciones experimentadas por las personas al momento en que se presentó el sismo. Como lo demuestra la gráfica, el reflejo de sobrevivencia se manifestó en el $33 \%$ de las personas, ya que declararon salir de su vivienda al momento de sentir el movimiento telúrico. En un sentido más individual, se tiene que el $26 \%$ se protegió, mientras que el $20 \%$ se quedó paralizado, lo que demuestra el impacto emocional, inesperado, del fenómeno. El sentido de solidaridad o de apego se manifestó en el 13 \% de los encuestados, ya que manifestaron que durante el sismo se protegieron a otras personas que se encontraban en la vivienda.

Finalmente, $8 \%$ de las personas comenzó a rezar, lo que indica que este sector de la población buscó consuelo o protección a partir de sus creencias religiosas. Estas reacciones frente al sismo son determinadas en la mayoría de los casos por la manera en que es percibido el riesgo. Por lo tanto, conocer la percepción es necesario, principalmente para "prever tipos de reacciones de la población y posibles formas de intervención para favorecer que los riesgos no evolucionen hasta convertirse en desastres" (González et al. 2013: 15). 
Gráfica 1. Respuesta de las personas durante el sismo

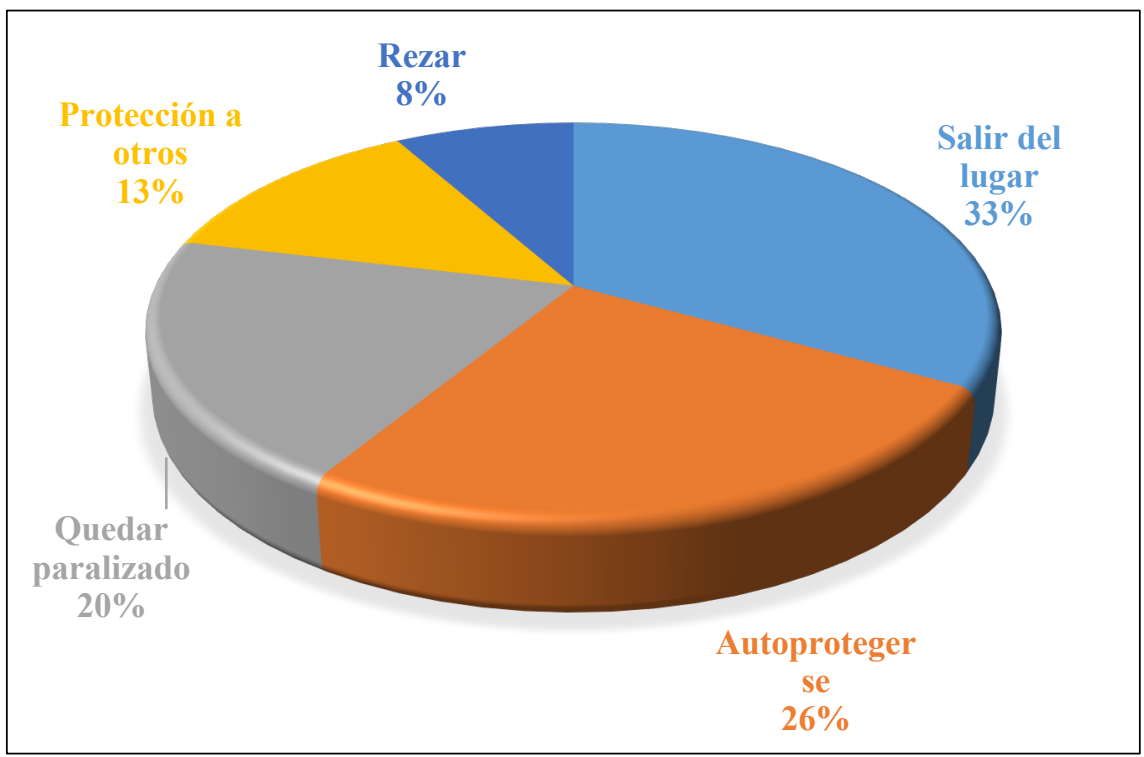

Fuente: elaboración propia, con base en De Dios (2020).

Una vez que el movimiento sísmico terminó, fueron diferentes las acciones que las personas decidieron emplear. En este caso, las acciones que la población realizó corresponden en un $38 \%$ salir de su vivienda, $34 \%$ se dedicó a comunicarse con su familia, 9 \% comenzó a escuchar los medios de comunicación para conocer la situación, 7 \% evaluó los daños que tenía su vivienda, $7 \%$ se dispuso a cortar el gas o electricidad y el $5 \%$ siguió con las actividades que se encontraba realizando. La respuesta social ante la emergencia es variada, pero se puede inferir que la población actúa en la medida de sus posibilidades para sentirse más seguro, ya sea en lo individual o a nivel familiar. Asimismo, empieza a poner en función sus redes sociales y familiares, las cuales son descritas como una forma de brindar apoyo de cualquier tipo como puede ser en el plano moral, económico, etcétera (Lomnitz, 1981, citada por Abello et al., 2011). Además, algunos de ellos comenzaron a verificar, en las fuentes de información, un posible desastre, ya sea para conocer su situación, la de otros miembros cercanos o del contexto más amplio: su ciudad. Ante este tipo de situaciones, Mileti y Sorensen (1990) mencionan que los mensajes deben provenir de emisores que la sociedad considere como creíbles.

Un dato que demuestra la disparidad de estas reacciones fue el bajo porcentaje de población que se dedicó a verificar los daños en su vivienda (7\%), ya que una de las primeras recomendaciones de los expertos en atención a desastres es verificar el estado de la vivienda para enfrentar posibles réplicas (Cenapred, 2014b). De igual modo, otro $7 \%$ de la población atendió las recomendaciones para cerrar las líneas de electricidad y gas para evitar daños colaterales asociados al sismo. Destaca también el $5 \%$ de los 
encuestados que declararon continuar con sus actividades cotidianas, lo que demuestra que las personas actúan en función a su propio sentido de seguridad en la vivienda.

\section{Percepción del riesgo sísmico}

El estado de Colima, además de su propensión a los sismos, también es susceptible a erupciones del volcán de Colima, ciclones tropicales, heladas, granizadas, torbellinos de baja escala, tormentas eléctricas, tsunamis, deslaves y, sumado a ellos, actividades antropogénicas como el traslado de material peligroso por carretera, presencia de un gasoducto y una regasificadora, así como las consecuencias asociadas a cada uno de estos fenómenos naturales y antropogénicos (Villanueva, et al., 2008). El conocimiento acerca de las amenazas a las que está expuesta la población es básica para la gestión del riesgo. En este caso, los fenómenos naturales que los entrevistados consideraron como los más peligrosos fueron, con un $63 \%$, los fenómenos geológicos, y un $37 \%$ manifestó que son los hidrometeorológicos.

$\mathrm{Al}$ ser una región propensa a la ocurrencia de fenómenos naturales y antropógenos, es necesario que la población esté consciente de las probabilidades que existen de que ocurra un sismo de gran magnitud, el cual puede estar asociado a otros eventos como explosiones, caída de infraestructura, etcétera. En este aspecto, el 74 \% de la población declaró que es muy probable que ocurra un sismo, mientras que el $19 \%$ cree que es algo que sucederá de manera inminente, y $7 \%$ mencionó que es poco probable que suceda. En ese sentido, la participación ciudadana dentro de la protección civil es fundamental en la prevención, ya que la población percibe de manera diferente el riesgo y está al tanto de cuáles son los principales riesgos que, en su contexto, pueden afectar tanto su integridad física como su patrimonio (Delgadillo, 1996).

Por su parte, lo que la población considera son las consecuencias que se derivan de un sismo, los derrumbes y daños a construcciones predominan con un $32 \%$, seguido de pérdidas humanas con el $28 \%$, destrucción con el $22 \%$, mientras que el resto considera elementos como afectaciones psicológicas, pobreza, inseguridad, escasez, pérdidas materiales y económicas, entre otras. De esta manera, cuando un sismo intenso se presenta, suelen detonar procesos de reconfiguración social, política y económica, pero principalmente, manifiesta las condiciones de riesgo y de vulnerabilidad con las que conviven los diferentes segmentos de una sociedad, aunado a éstos, altera la manera en que la sociedad percibe a la naturaleza (Esquivel, 2018; Colina y Ramírez, 1999; Morán, 2017).

Existen diversas causas por las que la población colimense cree que se originan los sismos. El $85 \%$ lo asocia a causas de origen geológico, ya sean por las placas tectónicas o bien por los volcanes; el 9 \% lo asocia al clima; el $3 \%$ desconoce su origen, mientras que el $2 \%$ le atribuye origen divino. 
Caballero (2007) hace mención de que, gracias a la consolidación del razonamiento científico en la era moderna, se empieza a comprender que los fenómenos, en su mayoría, son el resultado de procesos que se dan de manera natural y como consecuencia, se origina la idea de que los desastres son originados por la naturaleza y sus fenómenos, ocultando las razones sociales de los desastres.

En relación con el sentimiento de seguridad que manifestaron los encuestados, ésta se refirió a dos escalas, la vivienda y la ciudad. Los resultados arrojaron que el $68 \%$ consideró que su vivienda es segura ante los sismos, mientras que el $62 \%$ manifestó que su ciudad no es segura. ${ }^{3}$ En otras palabras, las personas se sienten más seguras en un entorno más familiar, más inmediato, en este caso su propia vivienda, ya que pueden tener un mayor control sobre las acciones que pueden poner en marcha para enfrentar un sismo, por ejemplo, reforzar algunas estructuras, ubicarse en el lugar más seguro, realizar una evacuación rápida, mantenerse informada sobre las instrucciones de las autoridades, etcétera. Por su parte, las personas que manifestaron que su ciudad no es segura (62\%), pueden asociar esta inseguridad a no conocer, de manera profunda, las condiciones previas para enfrentar los sismos, como puede ser el sustrato geológico donde se asienta la ciudad, la calidad de construcción de otras viviendas e infraestructura pública, etcétera.

En este sentido, cobra relevancia lo que propone Douglas (1996: 58) cuando comenta que "el individuo parece cortar la percepción de los riesgos altamente probables de manera que su mundo inmediato parece más seguro de lo que es en realidad". Por su parte, Wilches-Chaux (1993: 12-13), demostró con un ejemplo las prioridades de los individuos frente al riesgo, al establecer que "en una comunidad marginada asentada sobre una falla geológica activa en zona urbana, las prioridades no se referirán a estructuras sismo-resistentes ni a comités de emergencia que entren a actuar en caso de terremoto, sino al mejoramiento de la vivienda, la instalación de servicios públicos, la salud y nutrición básica y la organización de los miembros para obtener la solución a esas necesidades”. En ese sentido, el riesgo sísmico es demasiado cotidiano, sumado a que los sismos de gran magnitud forman parte del contexto colimense (González, 2009).

Por lo que respecta al sentimiento de miedo ante los sismos, la población consideró que los sismos son impredecibles (17\%) y no tienen control de lo que pueda suceder al momento de que éste ocurra. Una proporción del $14 \%$ asoció ese miedo a las experiencias pasadas, ya que de una manera u otra experimentaron momentos de pérdida, destrucción o angustia. Asimismo, el $24 \%$ relacionó su miedo a la capacidad destructiva y sus consecuencias, mientras que el $12 \%$ lo asoció a la intensidad de los movimientos, y el $11 \%$ siente temor por su integridad física y la de su familia. En este sentido, el

${ }^{3}$ En ocasiones las respuestas de los encuestados pueden ser contradictorias hasta cierto punto, ya que el $33 \%$ manifestó salir de su vivienda durante el sismo (Gráfica 1). 
miedo se puede fundamentar en dos elementos, el conocimiento técnico del fenómeno natural, debido a su potencialidad e impredecibilidad y, por el otro, a su experiencia previa a partir del sismo de 2003.

Por otro lado, se registraron expresiones de los encuestados a partir de la pregunta ¿para usted qué es un sismo?, y las palabras asociadas a estos. Se obtuvieron, mediante el conjunto SAM (análisis semántico del valor M, por sus siglas en inglés), la lista con las diez palabras con mayor puntuación, resultando que los sismos están asociados al movimiento, miedo, desastre, fenómeno natural, destrucción, placas tectónicas, pérdidas humanas, derrumbes, daños y pérdida. Los significados construidos por los encuestados son derivados de la respuesta que cada uno de ellos dio a la interrogante ¿qué es un sismo?, lo que permitió clasificarlos. En ese sentido, los significados se han clasificado a partir de la técnica de redes semánticas en cinco categorías: creencias y emociones, características del fenómeno, resultados postdesastre, componentes del desastre y respuesta a la emergencia. La Gráfica 2 muestra esta clasificación.

Gráfica 2. Categorías de significados según valor semántico

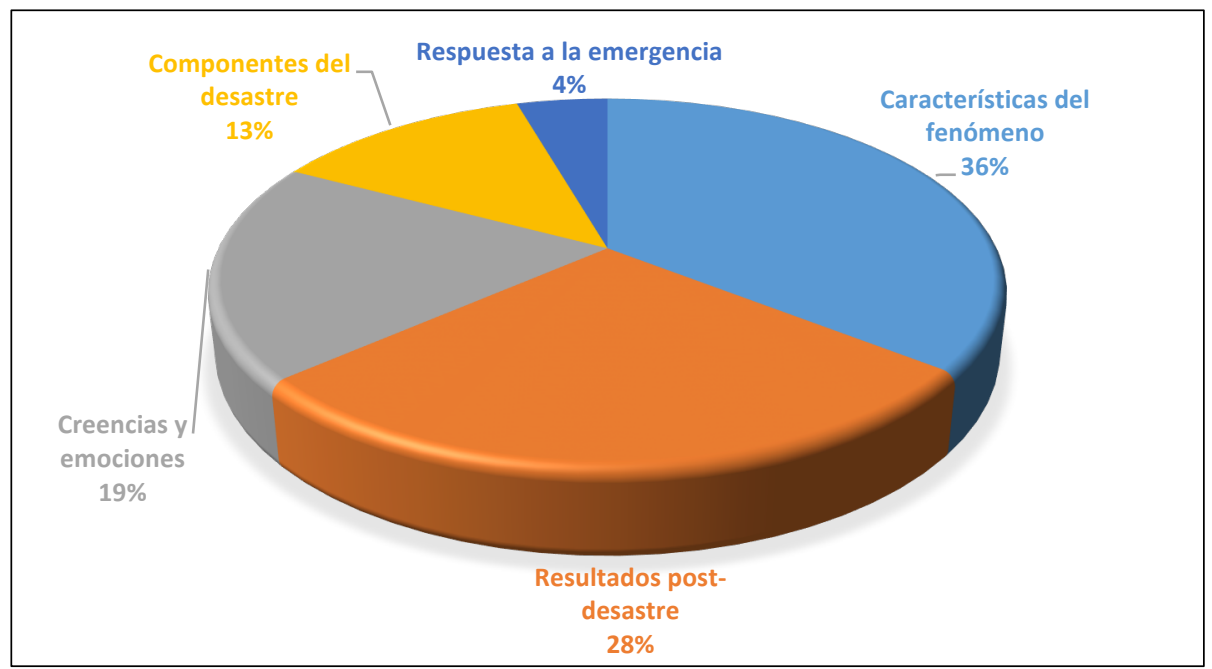

Fuente: elaboración propia, con base en De Dios (2020).

Las categorías en las que fueron agrupadas las palabras definidoras son los significados que se han construido a partir de sus experiencias al momento de la ocurrencia de los movimientos sísmicos y a los daños a los que están asociados. En este caso, la categoría de "creencias y emociones" contiene palabras como: angustia, ansiedad, desesperación, dios, incertidumbre, impotencia, estrés, miedo, preocupación, entre otras. La categoría de "características del fenómeno" incluye palabras como: clima, fuerza, actividad volcánica, geológico, placas tectónicas, energía, entre otras. Enseguida, la categoría de "daños asociados al desastre" incluyó palabras 
como: accidentes, destrucción, pérdida, escasez, damnificados, daños, incomunicación, derrumbes, entre otras más. Por otro lado, la categoría de "componentes del desastre" engloba las palabras: amenaza, emergencia, riesgo, desastre y peligro; mientras que la categoría de "respuesta a la emergencia" contiene palabras como: gritos, proteger, evacuar, llanto, correr, prevención, entre otras.

En este sentido, al cuestionar a los encuestados acerca de la palabra "sismo", el $34 \%$ de las expresiones estuvieron relacionadas con las características del fenómeno mismo, mientras que un $36 \%$ con las asociaciones léxicas, lo que indica una relación estrecha entre el significado "técnico" del término y el significado para esa porción de personas encuestadas. Esto demuestra que, de manera inmediata y sin razonarlo mucho, las personas se refieren primero a las características físicas que, para ellos, significa la palabra sismo, siendo éste el primer pensamiento que desarrollan en el recuerdo al momento en que se les cuestiona sobre este fenómeno geológico. Posteriormente, hacen referencia a los resultados del sismo, que pueden estar asociados a daños y pérdidas. En menor porcentaje se asociaron estas respuestas a creencias y emociones, componentes del desastre y, finalmente, a la respuesta durante la emergencia (Gráfica 3).

\section{Gráfica 3. Expresiones acerca de los sismos}

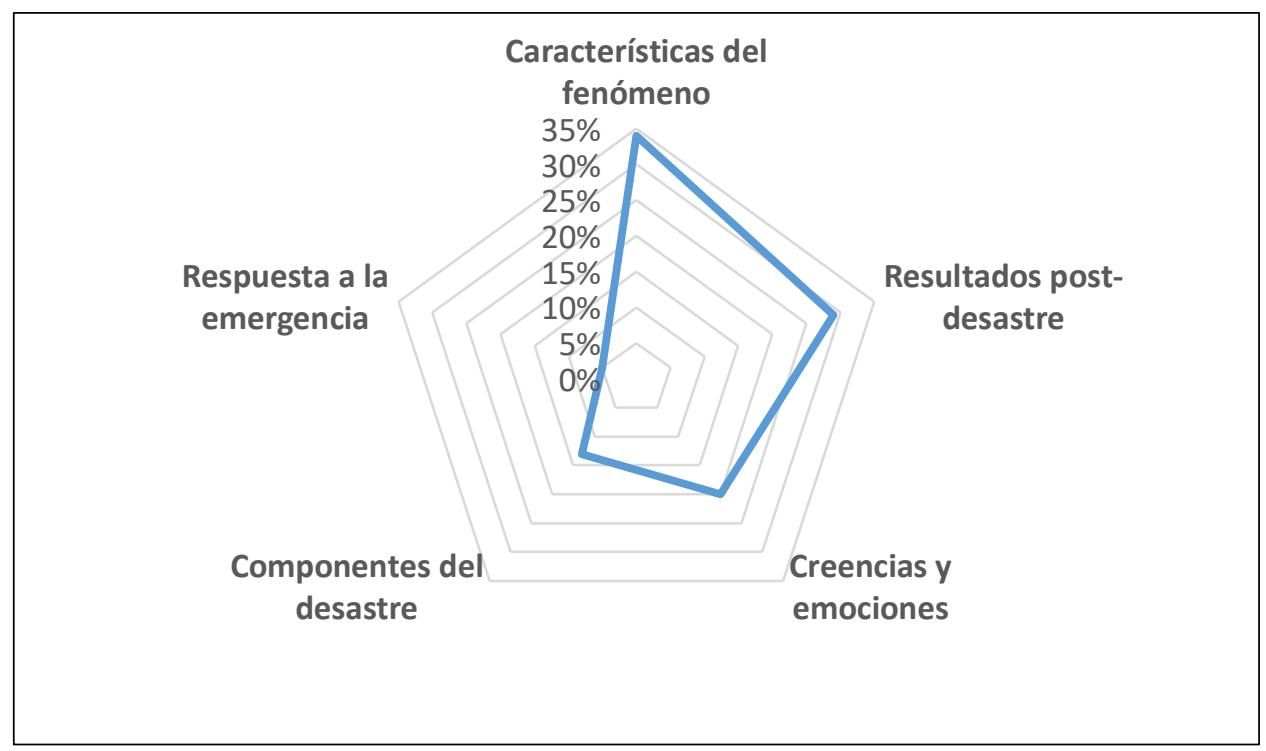

Fuente: elaboración propia, con base en De Dios (2020).

De la misma forma, en el tema de resultados postdesastre, el $29 \%$ de los encuestados los mencionó en sus expresiones, y en el $28 \%$ aparecieron en las asociaciones léxicas. Esto significa que los resultados del desastre o de la emergencia, son los que aparecen frecuentemente en el subconsciente cuando 
se piensa en estos movimientos telúricos sin ahondar mucho en el tema. Este bajo porcentaje en las respuestas puede estar asociado, quizás, al mismo proceso de olvido, o a que los daños registrados en su vivienda no fueron significativos para esa persona. Por lo tanto, los impactos del sismo, y su magnitud, determinarán en el futuro si se mantiene el recuerdo en la conciencia de la población. De igual modo, las creencias y las emociones presentan porcentajes de $20 \%$ con las expresiones y un $19 \%$ en las asociaciones léxicas, lo cual podría interpretarse como los recuerdos más próximos que se tienen sobre el sismo del 2003, los cuales mantienen una carga emocional latente.

En la categoría de los componentes del desastre, aparecen en un $13 \%$ de las expresiones, al igual que en las asociaciones léxicas, lo cual podría deberse a la implicación en cada una de las palabras que están dentro de esa categoría, las cuales hacen que venga a la mente la presencia de los riesgos a los que se encuentran expuestos, ya que reconocen dentro de su contexto, que los sismos representan un riesgo, pero ellos se sienten seguros en su vivienda. La última de las categorías es la respuesta a la emergencia, la cual aparece en un porcentaje muy bajo en ambas formas: $5 \%$ en las expresiones y $4 \%$ en las asociaciones léxicas, lo cual hace notar la carencia de capacidades para actuar ante un evento de esta magnitud. Esto pudiera deberse a diferentes factores como la inevitabilidad de los sismos, la carencia de un pronóstico o sistema de alerta temprana sobre sismos, como ocurre en el caso de los huracanes. Esto es un elemento central para incluirse en la gestión del riesgo sísmico a nivel local. La poca diferencia entre los porcentajes entre las expresiones y las asociaciones léxicas de las cinco categorías, puede estar asociada a que, tal y como lo expresan, le dan la misma relevancia a la palabra formulada.

De esta forma, profundizar en las actitudes frente a la amenaza sísmica, así como las reacciones durante y después de manifestado el sismo, ofrece herramientas que deben incluirse en la gestión del riesgo de desastres. Los insumos para la política de gestión del riesgo van en dos vías: por un lado, permite orientar un proceso de educación, capacitación o socialización del riesgo, donde se pueda complementar el conocimiento social (subjetivo) con el conocimiento científico (objetivo), y viceversa, acerca de las amenazas, el riesgo y la vulnerabilidad. Estos tres elementos, más la exposición al riesgo, forman parte del proceso que va conformando el riesgo de desastres, ya mencionado al inicio de este artículo.

Un elemento central en este proceso de socialización del riesgo, lo representan las propias reacciones de la población encuestada, ya que esto permitirá adecuar materiales, contenidos y estrategias para conocer el riesgo. Por otro lado, el diseño e implementación de planes o programas para reducir el riesgo de desastres, puede enriquecerse con las opiniones de la población en riesgo, donde las personas se vean reflejadas con sus aportaciones, conocimientos y experiencias para traducir esos instrumentos de política pública en guías para reducir el riesgo y ordenar el crecimiento 
de los asentamientos humanos. La participación social es un elemento clave en cualquier política para reducir el riesgo de desastres.

Finalmente, si se acepta la definición de gestión del riesgo de desastres como "el proceso sistemático de utilizar directrices administrativas, organizaciones, destrezas y capacidades operativas para ejecutar políticas y fortalecer las capacidades de afrontamiento, con el fin de reducir el impacto adverso de las amenazas naturales y la posibilidad de que ocurra un desastre" (EIRD, 2009: 19), también se debe reconocer, dentro de ese proceso, a la población en riesgo, la cual debe ocupar un papel más protagónico. Lo anterior se puede lograr reconociendo las actitudes y respuestas que aquí se analizaron, y no solamente la actuación de las autoridades federales, locales y de expertos en ese proceso, tal y como se viene realizando en la actualidad en la mayor parte de las estrategias oficiales de reducción del riesgo.

\section{Conclusiones}

Los sismos son eventos frecuentes en Colima. La historia sísmica es evidencia de que la zona es una región dinámica por la presencia de placas tectónicas que influyen en gran parte del territorio nacional. Sin embargo, no todos los sismos pueden estar asociados a desastres; para que esto ocurra, se debe combinar un evento intenso y una población vulnerable a ese tipo de fenómenos. Los instrumentos para una gestión del riesgo parten del conocimiento de las amenazas y del riesgo mismo, como lo es el Atlas de Peligros y Riesgos del Estado de Colima, documento aquí consultado.

En cuanto a la percepción del riesgo sísmico, "los riesgos percibidos son aquellos que los individuos consideran como reales a partir de sus experiencias y conocimiento del entorno" (Morán, 2010: 103). En el caso de la población colimense, destaca la relacionada a las características del fenómeno, demostrando que las personas asocian primero las características físicas al mencionar palabras como movimiento, fenómeno natural, placas tectónicas, fuerza, inesperado. De modo particular, algunas personas mencionaron otros fenómenos relacionados con los sismos como el clima, lo que indica que las personas pueden estar, o no, informadas acerca del origen de los sismos, cada una de ellas hace una interpretación de su realidad y, por lo tanto, del riesgo. Esto último denota que, aunque existe conocimiento sobre las características físicas del fenómeno, aún se puede ver cierto desconocimiento con el origen de éste, puesto que aún se asocia con la actividad del volcán de Colima, lo cual puede estar relacionado, pero los sismos de mayor magnitud han tenido su origen en el movimiento de las placas tectónicas.

Por otro lado, después de esta categoría se encuentran los resultados del desastre. Las palabras mencionadas en esta categoría fueron accidentes, destrucción, pérdidas humanas, escasez, problemas, damnificados, siniestro, daños, derrumbes, entre otras. El conjunto de estas palabras muestra que 
los resultados de un sismo como el ocurrido el 21 de enero de 2003 quedan dentro de la conciencia de la población, por lo que al pensar en un sismo suelen aparecer estas palabras dentro de su subconsciente. En la última de las categorías, respuesta a la emergencia, se dieron expresiones como calmarse, gritos, proteger, caos, llanto, reacción, correr, no correr, solidaridad, evacuar y prevención. Estas respuestas obedecen al instinto natural para responder ante una señal de peligro, lo cual representan formas rápidas e inmediatas que las personas ponen en marcha ante lo impredecible que es un sismo. La implementación de campañas de información, simulacros y otras estrategias podrían ayudar a que las personas puedan actuar de manera diferente para sentirse más seguras.

La percepción del riesgo de la población se constituye por una serie de factores que van desde la escala individual (significado sobre el sismo) hasta la social (respuesta a la emergencia), ya que el recuerdo del sismo sigue vigente. Este es un elemento que se debe recuperar para la implementación de políticas públicas orientadas a la gestión integral del riesgo de desastre sísmico, el cual puede operativizar cualquier estrategia que pretenda reducir el riesgo y, por lo tanto, manejar de mejor manera la emergencia y sus consecuencias. El involucramiento de las personas, a través del recuerdo que aún se tiene, sería un insumo más que garantizaría el éxito de cualquier intervención en esta materia. Las instituciones encargadas de reducir el riesgo de desastres deberán considerar este tipo de información.

Una de las principales limitaciones del trabajo radica en su corte temporal. Analizar la percepción del riesgo sísmico en Colima, justo después de haber ocurrido el sismo de 2003, debió de haber ofrecido opiniones diferentes a las aquí presentadas, ya que la experiencia y el recuerdo sería reciente. Afortunadamente, y después de 18 años de haber ocurrido este sismo, algunas personas narraron su experiencia con cierto detalle, guardando elementos de análisis que pueden ser retomados en estudios futuros, por lo que esta debilidad puede representar una fortaleza en la gestión del riesgo de desastres.

\section{Agradecimientos}

Agradecemos el apoyo del Conacyt por el otorgamiento de una beca de posgrado a la primera autora.

\section{Referencias}

Abello, Raimundo; Madariaga, Camilo, y Hoyos de los Ríos, Olga (1996). "Redes sociales: un mecanismo de supervivencia en sectores de pobreza". Investigación y Desarrollo, (4), pp. 73-89. Recuperado de 
http://rcientificas.uninorte.edu.co/index.php/investigacion/article/viewF ile/2578/1695

Alcántara-Ayala, Irasema; Garza, Mario; López, Alejandra; Magaña, Víctor; Oropeza, Oralia; Puente, Sergio; Rodríguez, Daniel; Lucatello, Simone; Ruiz, Naxhelli; Tena, Ricardo; Urzúa, Myriam, y Vázquez, Gloria (2019). "Gestión integral de riesgo de desastres en México: reflexiones, retos y propuestas de transformación de la política pública desde la academia". Investigaciones Geográficas, Instituto de Geografía-UNAM. DOI: 10.14350/rig.59784

Anzaldo, Mónica; Estrada, Jesús Salvador; Maisterrena, José Javier; Galindo, Andrés Ismael, y Ramos, Teresa de Jesús (2020). "Institución, autonomía y autogestión ante el desastre. (O de cómo los terremotos hacen temblar lo instituido)". Revista de El Colegio de San Luis. Nueva época, X(21), enero-diciembre, pp. 5-22. DOI: 10.21696/rcsl102120201097

Arellano, Aideé; Chávez, María Guadalupe, y Anguiano, Víctor Fernando (2012). "Vida cotidiana, problemáticas sociales y expectativas de vida en estudiantes de la Facultad de Letras y Comunicación de la Universidad de Colima, México. Exploración del significado social mediante Redes Semánticas Naturales (RSN)". Estudios sobre las Culturas Contemporáneas, 18(35), Época II, pp. 139-173.

Ayuntamiento de Colima (2012). Programa municipal de ordenamiento territorial de Colima. Sedesol. Recuperado de http://ipco.gob.mx/2019/index.php/marco-normativo/proyectos/2uncategorised/95-programa-municipal-de-ordenamiento-territorial-decolima

Banco Mundial (2012). Fonden: el Fondo de Desastres Naturales de MéxicoUna reseña. Washington, EUA: Banco Mundial/Banco Internacional de Reconstrucción y Fomento. Recuperado de http://www.proteccioncivil.gob.mx/work/models/ProteccionCivil/Almace $\underline{\text { n/fonden_resumen_ejecutivo.pdf }}$

Bracamontes, Beatriz (2015). "Vehículos de la memoria asociados con el sismo: y el desastre de 1941 en la ciudad de Colima, México". Estudios sobre las Culturas Contemporáneas, 21 (2), pp. 125-142. Recuperado de http://www.redalyc.org/articulo.oa?id=31639208007

Caballero, José Humberto (2007). "La percepción de los desastres. Algunos elementos desde la cultura". Gestión y Ambiente, 10(2), pp. 109-116. Recuperado de https://revistas.unal.edu.co/index.php/gestion/article/view/1416 
Cenapred (2003). Informe Técnico: El sismo de Tecomán del 21 de enero de 2003 (Me 7.6). México: Centro Nacional de Prevención de Desastres. Recuperado de http://www.proteccioncivil.gob.mx/work/models/ProteccionCivil/Resourc e/363/1/images/sismo tecoman.pdf

Cenapred (2004). Impacto socioeconómico de los principales desastres ocurridos en la República mexicana en el año 2003. México, D.F.: Centro Nacional de Prevención de Desastres. Recuperado de https://www.gob.mx/cenapred

Cenapred (2014a). Serie Fascículo: Sismos. Centro Nacional de Prevención de Desastres. México, D.F.: Centro Nacional de Prevención de Desastres. Recuperado de http://cenapred.gob.mx/es/Publicaciones/archivos/163FASCCULOSISMOS.PDF

Cenapred (2014b). Cartel de sismos. México, D.F.: Centro Nacional de Prevención de Desastres. Recuperado de http://www.cenapred.gob.mx/es/Publicaciones/archivos/221CARTELSISMOS.PDF

Cenapred (2020). Atlas nacional de riesgos. Atlas estatal de Colima. México, D.F.: Centro Nacional de Prevención de Desastres. Recuperado de http://www.atlasnacionalderiesgos.gob.mx/AtlasEstatales/?\&NOM EN $\underline{\mathrm{T}}=$ Colima\&CVE_ENT $=06$

Colina, Jaime y Ramírez de Alba, Horacio (1999). "Características de los sismos y sus efectos en las construcciones (Primera de dos partes)". CIENCIA ergo-sum, 6(1), pp. 83-89. Recuperado de https://dialnet.unirioja.es/servlet/articulo?codigo $=5139925$

Cortes, Esteban; Badillo Edgar, y Trejo, Jesús (22 de enero de 2003). "Tragedia: 21 muertos deja el terremoto". Diario de Colima. Recuperado de http://www1.ucol.mx/hemeroteca/pdfs/220103.pdf

Cuevas, Alicia y Seefoo, José Luis (2005). "Reubicación y desarticulación de La Yerbabuena. Entre el riesgo volcánico y la vulnerabilidad política”. Desacatos, (19), pp. 41-70. DOI: 10.29340/19.1044

Delgadillo, Javier (coord.) (1996). Desastres naturales: aspectos sociales para su prevención y tratamiento en México. México: Instituto de Investigaciones Económicas-UNAM, 292 pp.

De Dios, Karla Nayeli (2020). "La percepción y comunicación del riesgo sísmico: El sismo de 2003 en la ciudad de Colima” (Tesis de maestría en Administración Integral del Ambiente). Tijuana, B.C: El Colegio de la 
Frontera Norte/Centro de Investigación Científica y de Educación Superior de Ensenada, 101 pp.

Douglas, Mary (1987). "Les études de perception du risque: un état de l'art". En J. L. Fabián y J. Thies. La société vulnerable. Évaluer et maîtriser les risques. París: Presses de L'École Normale Supérieure.

Douglas, Mary (1996). La aceptabilidad del riesgo según las ciencias sociales, Barcelona, España: Paidós Studio.

Douglas, Mary y Wildavsky, Aarón (1982). Risk and Culture. An Essay on the Selection of Technological and Environmental Dangers. Berkeley: University of California Press, $224 \mathrm{pp}$.

EIRD (Estrategia Internacional para la Reducción de Desastres) (2009). Terminología sobre reducción del riesgo de desastre. Ginebra, Suiza: Estrategia Internacional para la Reducción de Desastres-Organización de las Naciones Unidas. Recuperado de https://www.unisdr.org/files/7817_UNISDRTerminologySpanish.pdf

Esquivel, Raúl (2018). "Algo de la ciencia e historia de los sismos y sus consecuencias". Revista Ciencia, 69(1), pp. 72-76. Recuperado de https://www.revistaciencia.amc.edu.mx/images/revista/69 1/PDF/Algod elacienciaehistoriadelossismos.pdf

Garza, Mario (1998). "Breve historia de la protección civil en México". En D. Garza-Salinas y M. Rodríguez-Velázquez (coords.). Los desastres en México, una perspectiva multidisciplinaria. México: Universidad Iberoamericana/UNAM/UAM-Xochimilco, pp. 246-287.

Gobierno del Estado de Colima (2015). Atlas de peligros y riesgos en el estado de Colima. Universidad de Colima. Recuperado de http://www.atlasnacionalderiesgos.gob.mx/AtlasEstatales/?\&NOM_EN $\underline{\mathrm{T}}=$ Colima\&CVE_ENT $=06$

González, Gabriela del Carmen (2009). "Inmunidad compartida ante el riesgo geológico en el estado de Colima, México". Language and Ecology, 3(1), pp. 1-14.

González, Gabriela del Carmen; Arellano, Aideé y Pérez, Alan (2013). Percepción del riesgo geológico en los jóvenes de la Universidad de Colima. México: Universidad de Colima, 104 pp.

Hernández, Roberto; Fernández, Carlos, y Baptista, María del Pilar (2014). Metodología de la investigación. México: McGraw Hill, 600 pp. 
Hinojosa, Guillermo (2008). "El tratamiento estadístico de las redes semánticas naturales”. SOCIOTAM, XVIII(1), pp. 133-154. Recuperado de https://www.redalyc.org/articulo.oa?id=654/65411190007

INEGI (2015). Encuesta Intercensal 2015. México: Instituto Nacional de Estadística, Geografía e Informática. Recuperado de https://www.inegi.org.mx/programas/intercensal/2015/

Mileti, Dennis y Sorensen, John (1990). Communication of Emergency Public Warnings: A Social Science Perspective and State-of-the-Art Assessment. EUA: U.S. Department of Energy, 162 pp. Recuperado de https://www.osti.gov/servlets/purl/6137387

Morán, Jorge Damián (2010). "Haciendo explícitos los riesgos del riesgo de desastre". Temas de Coyuntura, 61, julio de 2010, pp. 89-115. Recuperado de

http://revistasenlinea.saber.ucab.edu.ve/temas/index.php/temasdecoyun tura/article/view/1113

Morán, Jorge Damián (2017). Escenarios de riesgos y desastres por sismos e inundaciones en la zona metropolitana de la Ciudad de México. San Luis Potosí, México: El Colegio de San Luis, 279 pp.

Organización de las Naciones Unidas (2016). Informe del grupo de trabajo intergubernamental de expertos de composición abierta sobre indicadores y la terminología relacionados con la reducción del riesgo de desastres. Ginebra, Suiza. Asamblea General (resolución 69/284), Tema 19 c) del Programa Desarrollo sostenible: reducción del riesgo de desastres. Recuperado de http://comunidadpnacc.com/informe-del-grupo-detrabajo-intergubernamental-de-expertos-de-composicion-abierta-sobrelos-indicadores-y-la-terminologia-relacionados-con-la-reduccion-delriesgo-de-desastres/

Romo, Heriberto (1998). "La metodología de la encuesta”. En Jesús Galindo (coord.) Técnicas de investigación en sociedad, cultura y comunicación. México: Addison Wesley Longman, pp. 33-74.

SEDATU (Secretaría de Desarrollo Agrario, Territorial y Urbano) (2014). Atlas de riesgos naturales del municipio de Colima. México: Secretaría de Desarrollo Agrario, Territorial y Urbano. Recuperado de http://rmgir.proyectomesoamerica.org/PDFMunicipales/06002_COLIM $\underline{\text { A.pdf }}$

SEGOB (Secretaría de Gobernación) (2019). Recursos autorizados por declaratoria de desastre. México: Sistema Nacional de Protección CivilSecretaría de Gobernación. Recuperado de 
http://www.proteccioncivil.gob.mx/es/ProteccionCivil/Recursos_Autoriza dos_por_Declaratoria_de_Desastre

Slovic, Paul (1987). "Perception of Risk". Science, 236(4799), pp. 280-285. DOI: $10.1126 /$ science.3563507

Slovic, Paul (2000). The Perception of Risk. Reino Unido: Earthscan.

SSN (Servicio Sismológico Nacional) (2020). Catálogo de sismos. México: Servicio Sismológico Nacional/UNAM. Recuperado de http://www2.ssn.unam.mx:8080/catalogo

Vargas-Garduño, María de Lourdes; Méndez, Ana María, y Vargas, Alethia Danae (2014). "La técnica de las redes semánticas naturales modificadas y su utilidad en la investigación cualitativa”. Memorias académicas del IV Encuentro Latinoamericano de Metodología de las Ciencias Sociales. La investigación social ante desafios transnacionales: procesos globales, problemáticas emergentes y perspectivas de integración regional, del 27 al 29 de agosto de 2014. Heredia, Costa Rica. Recuperado de http://www.memoria.fahce.unlp.edu.ar/trab_eventos/ev.8204/ev.8204.pdf

Villanueva, Mauricio Javier; Cervantes, Oscar Salvador; Velarde, Oscar Eduardo, y Padilla, Raymundo (2008). "Base de datos de fenómenos naturales y desastres. Fondo Ada Rodríguez Zepeda (FARZ)". Memoria del IV Foro Colima y su Región: Arqueología, Antropología e Historia. Colima, 24 al 26 de julio de 2008. Recuperado de http://www.culturacolima.gob.mx/imagenes/foroscolima/4/25.pdf

Wilches-Chaux, Gustavo (1993). "La vulnerabilidad global". En Andrew Maskrey (comp.), Los desastres no son naturales. Red de Estudios Sociales en Prevención de Desastres en América Latina, pp. 11-44. Recuperado https://www.desenredando.org/public/libros/1993/ldnsn/LosDesastresNo SonNaturales-1.0.0.pdf

Zermeño, Ana Isabel; Arellano, Aideé Consuelo, y Ramírez, Vanesa Aidée (2005). "Redes semánticas naturales: técnica para representar los significados que los jóvenes tienen sobre televisión, Internet $\mathrm{y}$ expectativas de vida". Estudios sobre las Culturas Contemporáneas, XI(22), pp. 305-334. Recuperado de https://www.redalyc.org/articulo.oa?id=31602207

Editora asociada: Esperanza Tuñón Pablos

Recibido: 28 septiembre 2020

Aceptado: 9 febrero 2021 